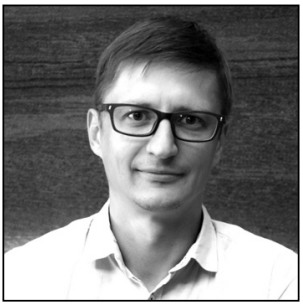

Ivo Pilving ${ }^{* 1}$

Vorsitzende der Verwaltungskammer, Staatsgericht

Dozent, Universität Tartu

\title{
Die Schaffung und Entwicklung der Verwaltungsgerichtsbarkeit in Estland
}

\section{Einleitung}

„Die Verwaltungsgerichtsbarkeit ist der Prüfstein des Rechtsstaats” betonte M. Stolleis im Oktober 2013 in Weimar, wo man den 150. Jahrestag der Errichtung des Bayerischen Verwaltungsgerichts gefeiert hat. ${ }^{{ }^{2}}$ Ungefähr zur gleichen Zeit fanden in Estland Veranstaltungen zum 20. Jahrestag des heutigen Justizwesens, u.a. der Schaffung von Verwaltungsgerichten, statt. Eigentlich besitzt das Verwaltungsgerichtsverfahren in Estland eine wesentlich längere Tradition, die fast 100 Jahre alt ist. In diesem Artikel versuche ich zu erläutern, aufgrund welcher, in der näheren und weiteren Vergangenheit gemachten Entscheidungen sich das heutige Modell der gerichtlichen Kontrolle des Verwaltungssystems entwickelt hat. Im Hauptteil des Artikels werden Wendepunkte der Entwicklungsgeschichte des estnischen Verwaltungsgerichtsverfahrens und Hauptmerkmale der Regulation des Verwaltungsprozesses in verschiedenen Epochen aus historischer Sicht unter die Lupe genommen (1). Dem folgt als Vergleichsmaterial eine kurze Charakterisierung des heutigen Systems, d.h. der Verwaltungsprozessordnung (Halduskohtumenetluse seadustik, VwGO) (2). ${ }^{*}$

\section{Entwicklungsstufen}

\subsection{Die erste Unabhängigkeitsperiode der Republik Estland}

Das aktuelle Modell der gerichtlichen Verwaltungskontrolle wurde in Estland nicht über Nacht erreicht. Es ist das Ergebnis einer langen, inzwischen abgebrochenen Evolution. Wurzeln des estnischen Verwaltungsprozesses reichen in die letzten Atemzüge des $^{*}{ }^{*}$ Russischen Kaiserreiches. Die Schaffung des

1 Der Artikel beruht auf dem Vortrag, der auf der Konverenz Academica „Modernse riigihalduse lätetel” (Anfänge der modernen Staatsverwaltung) (24.-25. Oktober 2013) in Tartu gehalten wurde. Ich bedanke mich bei Toomas Anepaio und bei Tõnu Anton für die nützlichen Gespräche, sowie bei Yoko Tees für die Übersetzung des Manuskripts.

2 M. Stolleis. Hundertundfünfzig Jahre Verwaltungsgerichtsbarkeit. - Deutsches Verwaltungsblatt 2013, S. 1274.

3 Siehe dazu W. Heermann. Auf Besuch in Tallinn und Helsinki. - BDVR-Rundschreiben, 2010/1, S. 10; I. Pilving. Als estnischer Verwaltungsrichter beim VG Freiburg. - Ibid., S. 11.

4 Das Territorium des heutigen Estlands gehörte bis zum Jahr 1917 zum Russischen Imperium, indem es Estland und einen Teil der Provinz Livland umfasste. Obwohl der zeitweilige Landrat der Provinz Estland schon am 28. Oktober 1917 eine Entscheidung über die hoheitliche Macht getroffen hat, gilt als Anfangsmoment der Republik Estland das in der Präambel des Grundgesetzes festgehaltene Datum der Ausrufung der Republik Estland - 24.2.1918. Siehe auch StGH 16.12.2013, 3-31-56-13, Rdn. 15. 
Verwaltungsgerichtsverfahrens war Anfang des 20. Jahrhunderts eines der Hauptziele der konstitutionellen Bewegung Russlands. ${ }^{*} 5$ In diesen Versuchen widerspiegelten sich größtenteils die sich in Deutschland verbreiteten Ideen über den Rechtsstaat und ihre Anwendungen: angefangen mit der Kantischen Philosophie bis zum Modell der deutschen Verwaltungsgerichte aus dem Jahr 1863, in dem das Justizwesen der Verwaltungsgerichte sowohl von der Exekutivgewalt als auch von allgemeinen Gerichten getrennt war. ${ }^{* 6}$ Im Russischen Kaiserreich konnte man die Verwaltungssachen aber nur im sog. System der Administrativ-Justiz lösen. ${ }^{*}$ In der ersten Instanz funktionierten als Administrativ-Justizorgane verschiedene Kommissionen für Staats-, Städte-, sog. Semstwo- als allgemeinen Landschafts- und Bauernangelegenheiten, Kommissionen für Fabriken- und Bergarbeitsangelegenheiten, Schulräte usw. - in der Regel in der Anwesenheit von Gouverneuren. In der zweiten Instanz funktionierten die sog. Administrativ-Abteilungen des Senats ${ }^{*}{ }^{*}{ }^{*}{ }^{9}$ Nach der Februarrevolution hat die provisorische Regierung von A. Kerenski am 31.5.1917 ein Dekret über die Prüfung der Verwaltungsangelegenheiten in Gerichten erlassen (Положеніе судахъ по административнымъ дъламъ). ${ }^{* 10}$ Die Inkorporation der Administrativgerichte in den allgemeinen Gerichten wurde initiiert, aber in Folge der bolschewistischen Oktoberrevolution wurde in Russland das ganze bisherige Jutizwesen liquidiert. ${ }^{* 11}$

In von Russland getrennten Estland hat die hiesige provisorische Regierung am 12.2.1919, d.h. noch vor der Fertigstellung und vor dem Inkrafttreten der Verfassung, eine Administrativgerichtordnung (AdmGO) eingeführt. ${ }^{*} 2$ Bis zum II. Weltkrieg wurde die Verwaltungsgerichtsbarkeit in Estland durch die „gewöhnlichen" Gerichte bzw. das allgemeine Gerichtssystem ausgeübt. Als erste Instanz fungierten von der Art der Sache abhängig Amtsrichter (früher Friedensrichter) und Bezirksgerichte (früher Friedensrichterplenen). Daher konnte man über Verwaltungsgerichte nur im funktionalen und nicht im organisatorischen Sinne sprechen. Die zweite und die höchste, in manchen Sachen (z.B. Klagen gegen Akten der Minister) die einzige zuständige Gerichtsinstanz war der Staatsgerichtshof (Riigikohus, StGH), in dem eine sog. Administrativabteilung oder Verwaltungsrechtskammer tätig war. ${ }^{*}{ }^{13}$ Im Jahr 1938 hatte die Administrativabteilung z.B. 688 Sachen zu lösen. ${ }^{*} 14$

AdmGO war inhaltlich eine Übersetzung aus dem Dekret der provisorischen Regierung von Kerenski aus dem Jahr 1917. In den in AdmGO nicht geregelten Angelegenheiten mussten ebenfalls die aus der russischen Zeit geltenden Normen der Zivilprozessordnung angewendet werden. ${ }^{*} 15$ A.-T. Kliimann kritisierte die Tatsache, dass AdmGO später nicht mit der Verfassung in Übereinstimmung gebracht wurde, besonders was die Zuständigkeit des Verwaltungsgerichts betraf. Die Zuständigkeit war von einer Seite durch kontrollierte Behörden, angefochtene Handlungen und Arbeitsbezirken der Behörden reguliert. ${ }^{* 16}$ War der

5 H. Oda. Judicial Review of Administration in the U.S.S.R. - Public Law 1989, S. 111; A. Palvadre. Tsiviilkohtu ja administratiivkohtu vahekorra korraldamine Tsiviilkohtupidamise seadustiku eelnõus [Die Gestaltung des Verhältnisses zwischen der Zivil- und Administrativgerichtsbarkeit im Entwurf der Zivilprozessordnung] (1933). - J. Erne (Hrsg.). Õigusteadlaste päevad 1922-1940. Protokollid [Rechtswissenschaftlertage 1922-1940. Protokolle]. Tallinn: Eesti Juristide Liit 2008, S. 695-696.

6 Vgl. Д. М. Чечот. Административная юстиция: теоретические проблемы [Administrativjustiz: theoretische Probleme]. Изд-во Ленингр. ун-та 1973, S. 8; M. Stolleis (Fn. 1), S. 1276.

7 Eine ähnliche Situation herrschte bekanntlich in Deutschland bis zum Jahr 1863, M. Stolleis (Fn. 1), S. 1275; D. Lorenz. Verwaltungsprozeßrecht. Berlin u.a.: Springer 2000, S. 8.

8 Sog. dirigierender Senat war das höchste Verwaltungs- und Gerichtsorgan des Kaiserreiches (auf Russisch: Правительствующий сенат).

9 H. Piip. Walitsemise-õigus: Riigivalitsemise sisemine korraldus. A. J. Jelistratovi ettelugemised [Verwaltungs-Recht: Die innere Organisation der Staatsverwaltung. Vorlesungen von A. J. Jelistratov]. Tallinn: Tallinna Eesti Kirj.-Üh. 1912, S. 47-51; Г. Е. Петухов. Административная юстиция в царской России [Administrativjustiz in Zarenrussland]. - Правоведение 1974/4, S. 73 .

10 Собрание узаконений и распоряжений правительства [Sammlung der Regierungserlasse und Verordnungen] 1917 I, 127, 692.

11 [K.] G[üldenstubbe]. Tsiviil- ning administratiivkohtute kompetentsi piiritamine [Die Abgrenzung der Zuständigkeiten der Zivil- und Administrativgerichte]. - Õigus 1929, S. 195, 196; H. Oda (Fn. 4).

12 Administratiivkohtukord. - Riigi Teataja (Staatsgesetzblatt - RT) 1919, 10, 23 mit später hinzugefügten Verbesserungen.

13 A.-T. Kliimann. Haldusprotsess [Verwaltungsprozess]. Tartu: Akadeemilise Kooperatiivi Kirjastus 1937, S. 85-92; P. Puusepp. Administratiivkohtu korraldus [Administrativgerichtsordnung] (1922). - J. Erne (Hrsg.) (Fn. 4), S. 52.

14 Kohtud 1938. aastal [Die Gerichte im Jahr 1938]. - Õigus 1939, S. 254, 261.

$15 \S \S 1$ und 44 AdmGO.

16 § 2 AdmGO; A.-T. Kliimann (Fn. 12), S. 65; s. noch: P. Puusepp (Fn. 12), S. 51; K. Güldenstubbe, Administratiiv- ja tsiviilkohtute allumuse piiritlemine [Die Abgrenzung der Administrativ- und Zivilgerichtsbarkeit] (1933). - J. Erne (Hrsg.) (Fn. 4), S. 701; K. Güldenstubbe (Fn. 10), S. 196. 
Beamte in der Norm nicht aufgelistet, musste man so lange innerhalb der Verwaltungshierarchie verklagen, bis man eine Entscheidung einer solchen Instanz erreichte, die im Gericht angefochten werden konnte. ${ }^{* 17}$ Auch die Regierung der Republik war nicht in dieser Liste. Der Rechtsschutz gegen die Akten der Regierung war möglich nur dann, wenn gegen ihre Umsetzung durch niedrigere Behörden eine Klage eingereicht wurde. ${ }^{* 18}$ Nach AdmGO konnten im heutigen Sinne sowohl Verwaltungsakten, Handlungen, Unterlassung als auch Verordnungen von allgemeiner Natur angefochten werden. ${ }^{*} 19$ Das Gesetz hat das Gericht bevollmächtigt, Verwaltungsakten aufzuheben, eine Verwaltung zu Handlungen zu verpflichten und eine Handlung zu verbieten sowie eine Gutmachung von Schäden und sogar eine Beseitigung von Beamten anzuordnen. Nach AdmGO konnten Personen sich zum Schutz ihrer persönlichen und finanziellen Interessen an das Gericht wenden. Ein Initiativrecht besaßen auch Aufsichtsbeamte. Dafür war eine spezielle Antragsart vorgesehen - ein Protest. ${ }^{* 20}$ Als Hauptaufgabe der gerichtlichen Kontrolle wurde allerdings der Schutz subjektiver öffentlicher Rechte angesehen. ${ }^{* 21}$

Im Verwaltungsgericht galt der Ermittlungsgrundsatz. ${ }^{{ }^{22}}$ Faktische Tatsachen sollten mit dem Stand des Zeitpunkts der Urteilsfällung ausgewertet werden, bei der Anwendung eines Gesetzes war der Zeitpunkt des Erlassens eines Verwaltungsaktes ausschlaggebend. ${ }^{*} 3$ Es fehlte eine klare Regelung und ein klares Verständnis über die Kontrolle des Ermessensspielraumes, jedoch gab es Beispiele über die Befriedigung von Klagen aufgrund des Ermessensmissbrauchs. ${ }^{*} 24$ Eine Gerichtssache konnte nur in einer mündlichen Verhandlung entschieden werden. ${ }^{*} 25$ Die Klage hatte keinen automatischen Suspensiveffekt, aber das Gericht hatte die Möglichkeit, die Wirksamkeit des angefochtenen Rechtsakts aufzuschieben. ${ }^{{ }^{2} 6}$

In dieser Periode war in rechtswissenschaftlichen Diskussionen über das Verwaltungsgericht eines von am meisten erörterten Problemen die Abgrenzung der Kompetenz von Verwaltungs- und Zivilgerichten, insbesondere die Frage des Präjudiz bzw. Vorentscheid: Kann das Gericht beim Lösen einer Verwaltungssache zivilrechtliche Tatsachen feststellen und umgekehrt, ob das Gericht bei der Lösung einer Zivilsache eine Möglichkeit zur Kontrolle der Rechtmäßigkeit von Entscheidungen der Exekutive hatte. Die herrschende Meinung verneinte in dieser Zeit erstaunlicherweise so eine präjudizielle Zuständigkeit der Verwaltungsgerichte, obwohl die gleichen Richter die Verwaltungssachen lösten, die auch in Zivilsachen zuständig waren. ${ }^{* 27}$ Die Verwaltungssachen mit einem eigentumsrechtlichen Bezug waren damals aber häufig, da den Großteil von gelösten Verwaltungssachen Bodenreformstreitigkeiten ausmachten, u.a. die Planung von Grundstücken aufgrund der von deutschbaltischen Gutsherren verstaatlichten Ländereien sowie die Benennung von Bauern auf die Grundstücke. ${ }^{* 28}$

17 J. Ramm. Adminisratiivkohtukord [Administrativgerichtsordnung]. Eraväljaanne 1931, § 2 Rdn. 1.

18 A.-T. Kliimann (Fn. 12), S. 140.

19 In der Regel konnte eine Verordnung nur zusammen mit für ihre Umsetzung gemachten Entscheidungen oder Handlungen angefochten werden, mit zusätzlichen Hinweisen auf Rechtssprechung. J. Ramm (Fn. 16), § 2 Rdn. 8-9, 12; A.-T. Kliimann (Fn. 12), S. 134, 137-140.

20 §§ 3, 22 AdmGO. Unter rechtlichen Interessen wurden subjektive Rechte gemeint, A.-T. Kliimann (Fn. 12 ), S. 297.

21 Vgl. P. Puusepp (Fn. 12), S. 50-51; L. Kanger, M. Leppik. Halduskohtu pädevus. Riigikohtu administratiivosakonna ja halduskolleegiumi praktika aastatel 1919-1940 ja 1992-2009 [Die Zuständigkeit des Verwaltungsgerichts. Die Praxis der Administrativabteilung und der Verwaltungsrechtskammer aus den Jahren 1919-1940 und 1992-2009]. - Juridica 2009/8, S. 508, 509 mit zusätzlichen Literaturangaben.

22 Das wurde sogar in drei Paragrafen betont, §§ 19-21 AdmGO.

23 A.-T. Kliimann (Fn. 12), S. 297.

24 A.-T. Kliimann, „Vaba kaalutlus” ja selle kohtulik kontrollimine [„Freies Ermessen” und seine gerichtliche Prüfung]. - Õigus 1928, S. 84, 89.

$25 \S 17$ AdmGO.

$26 \S 13$ AdmGO.

27 K. Güldenstubbe (Fn. 15), S. 702; A.-T. Kliimann. Tsiviilkohtu halduskohtulik eelmõist [Verwaltungsgerichtlicher Vorentscheid des Zivilgerichts]. - Õigus 1933, S. 289.

28 P. Puusepp. Maasaajate määramine ja administratiivkohus [Die Benennung der Siedler und Administrativgericht]. - Õigus 1922, S. 4 . 


\subsection{Die sowjetische Periode}

Nach der Annexion Estlands durch die Sowjetunion im Jahr 1940 wurde die gerichtliche Kontrolle über die Exekutivgewalt in Estland unterbrochen. Alle bisherigen Justizbehörden wurden aufgelöst. Zu dieser Zeit fehlte in der Sowjetunion praktisch ein gerichtlicher Schutz vor der Willkür der Beamten. Die Sowjetmacht an sich wurde als eine ausreichende Garantie für die Rechte des Proletariats gesehen. Wohl wurde Anfang der 1920er Jahre in Russland ein Gesetzentwurf zum Gesetz der Verwaltungsgerichte zusammengestellt und auch wurden thematische theoretische Publikationen publiziert. Mit dem Machtantritt Stalins wurde es aber endgültig klar, dass „es in der Sowjetunion für eine verwaltungsgerichtliche Klage keinen Platz gibt”. ${ }^{29}$ Nach dem Tod Stalins wurde die Kontrolle der Staatsanwaltschaft über die Rechtmäßigkeit der Verwaltung verstärkt, die Aufgabe dieses Apparats war jedoch nicht der Schutz von Rechten der Einzelnen. Nach dem damaligen Recht war eine gerichtliche Kontrolle nur bei einzelnen Arten von Verwaltungsstreitigkeiten möglich: vor allem in Verbindung mit administrativen Strafen für die Ordnungswidrigkeiten und Wählerlisten. ${ }^{*}{ }^{30}$ In Satellitenstaaten Jugoslawien und Bulgarien konnte man dagegen in derselben Periode eine wesentlich umfassendere gerichtliche Kontrolle einführen. Ähnliche Pläne konnten in Ungarn und in der Tschechoslowakei wegen der Invasion der Sowjetischen Streitkräfte nicht realisiert werden. Doch hat man auch in der Sowjetunion angefangen, theoretische Abhandlungen über die gerichtliche Kontrolle zu publizieren. ${ }^{*}{ }^{11}$ Ebenfalls war eine Erhöhung der Aktivität von Bürgern bei der Einreichung von Klagen an höhere Verwaltungsorgane und an den Parteiapparat ersichtlich. ${ }^{*}{ }^{32}$

Im Jahr 1977 wurde in der UdSSR eine neue Verfassung verabschiedet. § 58 Abs. 2 der Verfassung hat bestimmt: „Gegen Handlungen von Beamten, die gegen das Gesetz verstoßen, durch die die Vollmachten der Beamten überschritten oder die Rechte der Bürger eingeengt wurden, kann nach der gesetzlich festgelegten Regelung vor Gericht Einspruch erhoben werden." 33 Mit dem Absatz 2 des gleichen Paragrafen wurde auch das Recht zur Vergütung der durch die Staatsorgane und Beamten zugefügten Schäden gewährleistet. Wie viele andere in diesem Dokument gelistete Rechte ist auch das Grundrecht auf die Klage lange deklarativ geblieben. Eine vollständige Realisierung dieses Rechts wurde vor der Auflösung der Sowjetunion nicht erreicht. ${ }^{*} 4$ Zur Sicherung des Rechts, ein Gericht anzurufen hat man eine zeitaufwändige Zusammenstellung eines Gesetzesentwurfs vorgenommen: Über 10 Jahre wurden zum Beispiel theoretische Diskussionen darüber geführt, ob die Zuständigkeit des Verwaltungssachen lösenden Gerichts durch eine Generalklausel oder einer enumerative Aufzählung geregelt werden soll. Die Perestroika von Gorbatschow hat beträchtliche Fortschritte mit sich gebracht. Im XXVII. Kongress der Kommunistischen Partei (1986) hat man es für erforderlich gehalten, beschleunigt ein Gesetzespaket zur Demokratisierung der sowjetischen Gesellschaft zu verabschieden, u.a. die Regelung der Anrufung des Gerichts in Verwaltungssachen. Als Ergebnis davon verabschiedete der Oberste Sowjet der UdSSR am 30.6.1987 das Gesetz „Über die Regelung der Klageerhebung gegen die die Rechte von Bürgern einengende rechtswidrige Tätigkeit von Beamten”. ${ }^{*} 35$

Das Gesetz ist am 1.1.1988 in Kraft getreten. ${ }^{*}{ }^{6}$ Die Bürger haben das Recht erhalten, binnen eines Monats vor dem Volksgericht, d.h. vor dem allgemeinen Gericht eine Klage gegen Entscheidungen von Beamten zu erheben, wenn sie der Meinung waren, dass ihre Rechte verletzt wurden. Der Rechtsschutz wurde durch wichtige Einschränkungen begrenzt. Bei der Lösung der Sache konnte das Gericht die Entscheidung eines Beamten nur für rechtswidrig erklären und die Wiederherstellung der verletzten Rechte anordnen. Dem Gesetz über die Klageerhebung wurde nur in trivialen alltäglichen Streitigkeiten eine

29 H. Oda (Fn. 4), S. 111-121; vgl. J. Starilow. Gerichtliche Verwaltungskontrolle im System des russischen Staats- und Verwaltungsrechts. Speyer: Forschungsinst. für öffentliche Verwaltung 1999, S. 73, Fn. 74.

30 P. H. Solomon. Judicial Power in Russia: Through the Prism of Administrative Justice. - R. Sharlet, F. Feldbrugge (Hrsg.). Public Policy and Law in Russia: In Search of a Unified Legal and Political Space. Leiden: Martius Nijhoff Publishers 2005, S. 91. In 1986 hat V. Remnev jedoch 30 Kategorien von Rechtssachen aufgezählt, in denen eine Person gegen die Exekutivgewalt eine Klage im Gericht erheben konnte, Право жалобы и административная юстиция в CCCP [das Klagerecht und die Administrativjustiz in der UdSSR]. - Советское государство и право 1986/6, S. 22, 30.

31 Siehe insbesondere z.В. Д. М. Чечот (Fn. 5). Vgl. H. Oda (Fn. 4), S. 114; K. Merusk. Halduskohus ja kohtulik halduskontroll Eestis [Verwaltungsgericht und gerichtliche Prüfung der Verwaltung in Estland]. - Eesti Jurist 1992, S. 138.

32 В. И. Ремнев (Fn. 29), S. 23.

33 So auch im $\S 56$ der Verfassung der ESSR vom Jahr 1978.

34 K. Merusk (Fn. 30), S. 138.

35 NSVL Ülemnõukogu Teataja [Anzeiger des Obersten Rats der UdSSR] 1987, 26, 388.

36 H. Oda (Fn. 4), S. 115-117; В. И. Ремнев (Fn. 29), S. 22. 
praktische Bedeutung vorausgesagt. So beinhaltete die entsprechende Verordnung des Plenums des Obersten Gerichts der UdSSR vor allem praktische Hinweise zur Lösung der Streitigkeiten bei der Anmeldung vom Wohnort, von Eigenheimen und Fahrzeugen sowie bei der Einweisung in Krankenhäuser. ${ }^{*} 7$ Da es in der UdSSR praktisch keine frühere Tradition der gerichtlichen Kontrolle der Verwaltung gab, ist ganz verständlich, dass man die Verwaltungssachen mit Vorsicht betrachtet hat und dass man der Erneuerung keinen schnellen Erfolg vorhergesagt hat. Aufgrund des Drucks vom Parteiapparat galt diese in dem Gesetz beschriebene gerichtliche Kontrolle nicht für Entscheidungen aller kollegialen Organe und betraf auch nicht die Fragen, die einen Bezug auf die staatliche Sicherheit und Landesverteidigung hatten. Im höher gestellten Verwaltungsorgan wurde ein verbindliches Vorverfahren eingeführt. Die Möglichkeit, ein Rechtsbehelf gegen ein Urteil einzureichen wurde ausgeschlossen, die Verwaltungssachen konnten nur durch einen Überwachungsprotest eines Staatsanwalts in ein höher gestelltes Gericht gelangen. Das verbindliche Vorverfahren und die Begrenzung des Rechts auf Rechtsbehelfe wurden aufgrund der Kritik von Abgeordneten des Obersten Sowjets ziemlich bald aufgehoben (20.10.1988). Schon am 2.11.1989 hat der Oberste Sowjet der UdSSR eine erneute Redaktion des Gesetzes über die Erhebung der Klage verabschiedet. Auch seine Überschrift ${ }^{*} 38$ bestätigte, dass ab jetzt auch das Verklagen die kollegialen Organe ermöglicht wird (§ 2). Wohl wurde aber das Verklangen von „Akten normativer Art” ausgeschlossen (§ 3).

Obwohl es interessant wäre, die weitere Entwicklung des Verwaltungsgerichtsverfahrens in Russland zu folgen, ${ }^{*} 39$ muss man zugeben, dass es ab dem Mai 1990 Estland nicht mehr betrifft. Am 30.3.1990 hat der Oberste Sowjet Estlands eine Übergangszeit für die Wiedererlangung der staatlichen Unabhängigkeit verkündet. Die Staats-, Verwaltungs-, Gerichts- und Staatsanwaltschaftsorgane Estlands wurden vom System der UdSSR getrennt. ${ }^{* 0} \mathrm{Zu}$ diesem Zeitpunkt hat man auch in anderen Staaten des Ostblocks angefangen, die gerichtliche Kontrolle zu verstärken und einzuführen. ${ }^{*}{ }^{4}$

\section{3. Übergangszeit und Gerichtsreform}

Noch vor der Übergangszeit wurde in Estland die einigermaßen liberalere Fassung der Verfahrensordnung der UdSSR aus dem Jahr 1988 über die Klageerhebung übernommen. Es erfolgte durch die Ergänzung der Zivilprozessordnung der ESSR (ZPO der ESSR) ${ }^{*}{ }^{2}$ - die Verwaltungssachen wurden in Volksgerichten als Zivilsachen besonderer Art behandelt. Das Gesetz der UdSSR zur Unterwerfung der kollegialen Organe unter die gerichtliche Kontrolle hat man in Estland nicht in Kraft setzen können. Stattdessen wurde die Zuständigkeit der Gerichte durch zersplitterte Sondergesetze erweitert, z.B. die Gemeindeordnung, das Unternehmensgesetz, das Gesetz über Grundsätze der Eigentumsreform. ${ }^{*} 4$ Die Volksgerichte mussten auch Klagen gegen die sog. Verwaltungsstrafen der Administrativkommissionen, d.h. im heutigen Sinne die Fälle von Ordnungswidrigkeiten lösen. ${ }^{*} 4$ Das Verwaltungsrecht hat damals hauptsächlich das Bestrafen von sog. Verwaltungsrechtsverletzungen bedeutet. Die dem westeuropäischen Verständnis entsprechenden Verwaltungsstreitigkeiten bildeten Anfang 1990er Jahre nur einen unwichtigen Teil von der Arbeit der Volksgerichte. Insgesamt hat man im Jahr 1988 in Estland 13.335 Zivilsachen gelöst, wobei 225 davon die „vom Administrativrecht hervorgehenden Rechtssachen” waren. 1989 waren die entsprechenden Werte 11002 und $171 .{ }^{*} 45$

37 Die Verordnung Nr. 15 vom 23.12.1988 des Plenums des Obersten Gerichts der UdSSR.

38 Gesetz über die Regelung der Klageerhebung gegen die die Rechte von Bürgern einengende rechtswidrige Tätigkeit von staatlichen Behörden und Beamten, NSVL Rahvasaadikute Kongressi ja Ülemnõukogu Teataja (Anzeiger der Volksabgeordnetenversammlung und des Obersten Rats der UdSSR] 1989, 22, 416.

39 Jetzt werden Verwaltungssachen in Russland von allgemeinen Gerichten, im Wirtschaftsbereich auch von der Arbitrage gelöst. Seit dem Jahr 1993 gilt das „Gesetz über die gegen die Beamten eingereichten Klagen”. S. P. H. Solomon (Fn. 29), S. 85; J. Starilow (Fn. 28), S. 53-65.

40 Das Gesetz des Obersten Sowjets der Republik Estland „Eesti valitsemise ajutise korra alustest” (Über die Grundlagen der zeitweiligen Regelung des Regierens über Estland), 16.5.1990.

41 Siehe z.B. das Gesetz der DDR vom 1.7.1989 über die Nachprüfung von Verwaltungsentscheidungen, siehe O. Kringe. Gerichtlicher Rechtsschutz gegen Verwaltungsentscheidungen in der DDR. - Neue Juristische Wochenschrift 1989, S. 2450; D. Lorenz (Fn. 6), S. 14.

42 Das Gesetz des Präsidiums des Obersten Sowjets der ESSR vom 23.6.1988, § $244^{1} \mathrm{ff}$.

43 K. Merusk (Fn. 30), S. 142.

44 § 236 Abs. 1 Nr. 2, §§ 241-244 ZPO; § 297 des Ordunguswidrigkeitengesetzes.

45 J. Odar. Tsiviilasjad kohtutes 1989. a. [Die Zivilsachen in Gerichten im J. 1989]. - Eesti Jurist 1990, S. 187. 
Während der Übergangsperiode fanden Diskussionen sowohl über die Einführung einer neuen Verfassung als auch über die Umstrukturierung aller Zweige der Staatsmacht statt, dies umfasste auch die Gerichtsreform. Auf dem Tisch waren Ideen von der Wiedereinführung vom Gerichtssystem der ersten Unabhängigkeitszeit bis zur Bildung von Schwurgerichten. ${ }^{*} 46$ In der Praxis sollte man etwas mit sog. genossenschaftlichen Gerichten, der Arbitrage und mit Ordnungswidrigkeiten lösenden Administrativkommissionen vornehmen, ${ }^{*} 47$ auch hat die Zunahme der Zahl von Ordnungswidrigkeitssachen Sorgen gemacht. Das Recht der UdSSR hat für das Auseinandersetzen mit diesen Sachen Administrativ- und Vollstreckungsrichtern an Volksgerichten vorgesehen. ${ }^{*} 48$ Als eine Lösung wurde die Möglichkeit vorgeschlagen, die Stellen von Verwaltungsrichtern auch an Land- und Stadtgerichten zu schaffen, die als Ergebnis der Gerichtsreform ihre Tätigkeit aufnehmen sollten, wobei langfristig nicht ausgeschlossen wurde, dass in größeren Städten aufgrund der Administrativrichter auch Verwaltungsgerichte gebildet werden. Man überlegte sowohl die Bildung einer Verwaltungskammer am obersten Gericht als auch die Einrichtung eines gesonderten höheren Verwaltungsgerichts nach dem finnischen und schwedischen Vorbild. Vor allem wollte man aber die Zivilrichter von der stark zunehmenden verwaltungsrechtlichen Nebentätigkeit befreien. ${ }^{*}{ }^{*} 9$ Als rechtliche Grundlage der Gerichtsreform wurde am 23.10.1991, d.h. nach der Wiedererlangung der Unabhängigkeit, aber vor der Verabschiedung der Verfassung, das Gerichtsverfassungsgesetz der Republik Estland (GVG) verabschiedet. ${ }^{*}{ }^{0}$ Das hat nach dem obengenannten Vorschlag an allgemeinen Gerichten (Land- und Stadtgerichten) tätige Verwaltungsrichter und in größeren Städten separate Verwaltungsgerichte vorgesehen. Die Verwaltungsrichter und -gerichte sollten vor allem Ordnungswidrigkeitssachen lösen (§ 19 GVG). Doch hat man in den Motiven zum Gesetzesentwurf eine gewisse Unwissenheit über die Entwicklung des Verwaltungsprozesses in nächsten Jahren ausgedrückt. ${ }^{*}{ }^{1}$ Die klassischen Verwaltungssachen wurden mit dem Gesetz vom 1991 doch in die Zuständigkeit keiner Gerichte gegeben, die Zuständigkeit lag im Ermessen des Justizministers (§ 17 Abs. 2). ${ }^{*} 52$ K. Merusk z.B. hat diese Situation scharf kritisiert, indem er aufgerufen hat, die Verwaltungsgerichte nach westeuropäischen Traditionen zu schaffen und sie von der Bearbeitung von Ordnungswidrigkeiten zu befreien. ${ }^{*} 3$

Das am 29.6.1992 durch Volksabstimmung verabschiedete Grundgesetz hat das Klagerecht eines Einzelnen (§ 15) und die im Gerichtsverfassungsgesetz vom 1991 vorgeschriebene Errichtung von Verwaltungsgerichten (§§ 148 Abs. 1 Zf. 1, 149 Abs. 1) gewährleistet, ohne ihre Aufgaben zu präzisieren. Im Rahmen der Anwendung von GVG wurden in Tallinn, Tartu und Narva die Verwaltungsgerichte errichtet. In anderen Bezirken wurden an Land- und Stadtgerichten die Stellen der Verwaltungsrichter geschaffen. ${ }^{*} 4$ Im September 1993 nahmen in Verwaltungsgerichten 13, in Land- und Stadtgerichten 23 Verwaltungsrichter ihre Arbeit auf. Die Verwaltungsrichter wurden als „niedriger gestellte Richter” angesehen. Insbesondere kam es im Gehalt von Verwaltungsrichtern zum Ausdruck, das im Vergleich zu Gehältern anderer Richter der ersten Instanz bis zu 10\% niedriger sein konnte. ${ }^{*} 55$

46 J. Raidla. Teel kriisist õigusriiki [Auf dem Weg von der Krise in den Rechtsstaat]. - Eesti Jurist 1990, S. 182, 185; J. Odar. Eesti kohtukorralduse kujundamine [Die Gestaltung des Gerichtssystems Estlands]. - Eesti Jurist 1990, S. 100, 103; Erläuterung zum Entwurf der Gerichtsverfassung der Republik Estland und des Gesetzes über die Stellung der Richter der Republik Estland. - Staatsarchiv Estland, ERA.R-3.3.15918 (1991), S. 3.

47 J. Kirikal. Kohtureform ja põhiseadus [Gerichtsreform und Verfassung]. - Eesti Jurist 1990, S. 251, 252-253; M. Rask. Ees on kohtureform [Es steht die Gerichtsreform vor]. - Eesti Jurist 1990, S. 318, 319.

$48 \S \S 5,18$ der Grundsätze der Gerichtsverfassung der UdSSR und Sowjetrepubliken - RKÜT 1989, 23, 441.

49 J. Odar (Fn. 45), 103; J. Odar. Kolmeastmelise kohtusüsteemi kujundamine ja kohtusüsteemi lülid [Die Gestaltung und Glieder des dreistufigen Gerichtsssytems]. - Eesti Jurist 1991, S. 329, 330-331; J. Kirikal (Fn. 46), S. 254, die Einrichtung der gerichtlichen Kontrolle über die Tätigkeit der exekutiven und organisatorischen Behörden und Staatsbeamten wurde als ein „sehr sensibles und schmerzhaftes” Thema bezeichnet.

$50 \quad$ RT 1991, 38, 472.

51 Erläuterung (Fn. 45), S. 6; § 42 des Gesetzes zur Veränderung und Ergänzung des am 16.12.1992 verabschiedeten „Gerichtsverfassungsgesetzes der Republik Estland” und „Gesetzes über die Stellung der Richterder der Republik Estland”. Siehe auch: U. Lõhmus. - Ü. Madise, B. Aaviksoo u.a. (Hrsg.). Eesti Vabariigi põhiseadus. Kommenteeritud väljaanne [Die Verfassung der Republik Estland. Kommentierte Ausgabe]. Tallinn: Juura 2012, § 148 Komm. 5; vgl. der Vortrag von J. Adams. - die erste Lesung des VwGO-Entwurfes 9.6.1993. VII Riigikogu 191 SE, http://www.riigikogu.ee/.

$52 \quad$ K. Merusk (Fn. 30), S. 138.

53 Ibid., S. 144; s. auch: Vortrag von K. Tamme - die erste Lesung des VwGO-Entwurfes (Fn. 50).

54 §§ 2-4 des Gesetzes über die Festlegung der Anzahl von Gerichten der Republik Estland sowie ihrer Zusammensetzung und der Anzahl von Beisitzern der Land- und Stadtgerichte. - RT 1993, 1, 1.

55 Eesti Vabariigi kohtuniku staatuse seadus (Gesetz über die Stellung der Richter der Republik Estland), § 31 Abs. 4 - RT 1991, 38,473 . 
In der zweiten Instanz begannen die Bezirksgerichte und in der Kassationsinstanz der Staatsgerichtshof die Verwaltungssachen zu bearbeiten. ${ }^{*}{ }^{6}$ Dabei ist die Zuständigkeit des Staatsgerichtshofs begrenzt, er überprüft die Entscheidungen eines Bezirksgerichts nur aus dem Standpunkt der richtigen Anwendung des materiellen Rechts und der Einhaltung von Verfahrensvorschriften. ${ }^{*} 57$

\subsection{Verwaltungsgerichtsordnung vom Jahr 1993}

Neben dem organisatorischen Aufbau der Gerichte brauchte man für das Starten der Arbeit von Verwaltungsgerichten Verfahrensvorschriften. Neben dem Gesetz über Ordnungswidrigkeiten, ${ }^{,}{ }^{58}$ das die Überprüfung und die Verhängung von Verwaltungsstrafen im Verwaltungsgericht regelte, beschloss man ein Gesetz zur Lösung von nicht strafbaren Verwaltungsstreitigkeiten auszuarbeiten. Der Gesetzesentwurf, der anfangs den Titel „Gesetz über Lösung von Verwaltungsstreitigkeiten” trug, wurde am 21.6.1993 unter dem Namen Verwaltungsprozessordnung verabschiedet. ${ }^{*} 9$ Nach der Erläuterung des Gesetzesentwurfs hat die den Entwurf ausgearbeitete Arbeitsgruppe bis zum Jahr 1940 gegoltene AdmGO als Grundlage genommen und den aktuellen Bedingungen angepasst. ${ }^{* 60}$

VwGO vom Jahr 1993 war in mehreren Aspekten AdmGO ähnlich, aber auch der Einfluss von ZPO der ESSR war eindeutig zu sehen. Die Zuständigkeit des Verwaltungsgerichts wurde durch die Kombination einer Generalklausel und zwei Sonderregelungen von $\S \S 3$ und 4 (Art der Verwaltungstätigkeit sowie zu überprüfende Institutionen und Behörden) bestimmt. ${ }^{*} 1$ Es konnten einzelne Verwaltungsakten, Handlungen und Verwaltungsverträge angefochten werden, im Gesetz wurden gesondert Entscheidungen der Wahlkommissionen genannt. ${ }^{*} 62$ Das Gesetz versuchte die Behörden und Beamten aufzulisten, die der Kontrolle des Verwaltungsgerichts unterworfen sind. Im Gegensatz zu § 2 AdmGO hatte diese Liste keine den Rechtsschutz begrenzende Bedeutung mehr, weil man sich an das Verwaltungsgericht auch zur Lösung aller anderen öffentlich-rechtlichen Streitigkeiten wenden konnte, für die das Gesetz keinen anderen Rechtsweg vorgesehen hat. ${ }^{*} 63$ Nach dem Gesetz sollten außerhalb der Zuständigkeit der Verwaltungsgerichte solche Klagen bleiben, deren Lösung mit einer zivilrechtlichen Streitigkeit verbunden war. ${ }^{* 64}$ Schon in ersten Verwaltungssachen hat der wieder seine Tätigkeit aufgenommener Staatsgerichtshof erläutert, dass zum Ausschluss der Zuständigkeit des Verwaltungsgerichtes nicht jede Verbindung mit dem Zivilrecht ausreichend ist, sondern die angefochtene Verwaltungshandlung muss in einem zivilrechtlichen Verhältnis stattgefunden haben. ${ }^{*} 65$ Dies hat wesentlich das Lösen der im Rahmen der erneuten Eigentumsreform entstandenen zahlreichen Streitigkeiten vereinfacht. Die Staatshaftungsansprüche und die Klagen in Bezug auf privatrechtliche Geschäfte der Verwaltung mussten damals ausnahmslos im Zivilverfahren behandelt werden. ${ }^{* 66}$

Nach VwGO 1993 waren solche Personen klagebefugt, deren Rechte ein Verwaltungsakt oder eine Handlung verletzt hat. Im Vergleich zu ZPO der ESSR hatten Vereinigungen von Personen zum Schutz ihrer Mitglieder oder anderer Personen kein allgemeines Klagerecht mehr. ${ }^{* 67}$ So eine Möglichkeit konnte nur durch ein Sondergesetz geschaffen werden. VwGO 1993 hat zugleich ähnlich zu AdmGO den Aufsichtsbeamten eine Möglichkeit zur Protesteinreichung gegeben. Die Klagefrist wurde auf einen Monat gesetzt, bemerkenswerterweise war ein Protest gänzlich fristlos. Es gab kein allgemeines verbindliches Vorverfahren,

\footnotetext{
$\S \S 148$ und 149 der Verfassung.

Angefangen mit dem § 40 Abs. 1 von VwGO aus dem Jahr 1993 bis zu § 229 Abs. 1-3 von jetzt gültigen VwGO.

In Kraft getreten am 1.8.1992 - RT 1992, 29, 396.

59 RT I 1993, 50, 694; ist am 15.9.2003 zusammen mit der Zivilprozessordnung sowie mit der Strafprozessordnung der Berufung und Kassatsion in Kraft getreten ( $\$ 74$ VwGO a.F.).

60 Erläuterung zum Entwurf der Verwaltungsprozessordnung. - VII Riigikogu, SE 191 I.

61 K. Merusk. Halduskohtu mõiste, organisatsioon ja pädevus haldusvaidluste lahendamisel [Begriff, Organisation und Zuständigkeit des Verwaltungsgerichts bei der Entscheidung über die Verwaltungsstreitigkeiten]. - Juridica 1994/2, S. 47, 48.

62 So auch in $\S \S 238-240$ ZPO der ESSR.

63 z.B. StGH 3.11.1995, III-3/1-32/95; K. Merusk (Fn. 60), S. 47-49.

$64 \S 3$ Abs. 2 Nr. 1 VwGO 1993.

65 StGH 23.2.1994, III-3/1-2/94; K. Merusk (Fn. 60), S. 50. Vgl. oben, Fn. 26.

66 K. Merusk, I. Koolmeister. Haldusõigus [Verwaltungsrecht]. Tallinn: Juura 1995, S. 125.

67 Vgl. § $244^{1}$ Abs. 2 ZPO der ESSR. StGH hat auch schon früh betont, dass die Rechtmäßigkeit von Verwaltungshandlungen an sich keine Grundlage für eine Klageerhebung ist, siehe StGH 31.3.1995, III-3/1-4/95.
} 
durch Sondergesetze war seine Einrichtung möglich. ${ }^{* 68}$ Gerichtssache konnte nur in einer Sitzung gelöst werden $^{* 69}$ und die Sitzung sollte innerhalb eines Monats ab der Klageerhebung gehalten werden. ${ }^{*}{ }^{70}$ Bei der Prüfung der Sache sollte man vom Ermittlungsgrundsatz ausgehen. ${ }^{* 71}$ Obwohl in der Literatur die Meinung geäußert wurde, dass das Gericht faktische Tatsachen mit dem Stand des Zeitpunktes der Fällung des Urteils feststellen muss, ${ }^{*} 72$ ist in der Praxis die ex post Betrachtungsweise die Regel geworden - als Grundlage wurden die Tatsachen im Moment des Erlassens der Verwaltungsaktes genommen. ${ }^{*}{ }^{3}$ Die Befugnisse vom Verwaltungsgericht waren in den 1990er Jahren begrenzt. Das Gericht konnte einen Akt oder eine Handlung nur für rechtswidrig erklären und gleichzeitig der Verwaltung einen „Vorschlag” unterbreiten, die Sache nochmals zu bearbeiten. ${ }^{*} 74$ Der Vorschlag wurde jedoch als rechtlich verbindlich angesehen. ${ }^{*} 75$

Die Rahmenbedingungen für die Tätigkeit der Verwaltungsgerichte waren in 1990er Jahren kompliziert. Es gab keine Gesetze für den allgemeinen Teil des Verwaltungsrechts, es gab kaum Rechtsprechung und Fachliteratur. Die Tätigkeit der Institutionen der Exekutive war durch zersplitterte sektorale Rechtsakte reguliert, oftmals nur durch Verordnungen oder Verwaltungsvorschriften, oft gab es Widersprüche zur Verfassung. ${ }^{*} 6$ Die dem Verwaltungsverfahren zu stellenden Anforderungen mussten aufgrund der Verfassung und der Erfahrungen anderer Staaten geschaffen werden. Vor allem in Sachen der Eigentumsreform hat der Staatsgerichtshof wichtige Grundsätze für die weitere Entwicklung des allgemeinen Verwaltungsrechts und des Verwaltungsprozessrechts gebildet: Begründungspflicht von Verwaltungsakten, ${ }^{*} 77$ die Grenzen der Kontrolle von Ermessensentscheidungen der Verwaltung, ${ }^{*}{ }^{8}$ Fragen der Veränderung eines früheren Verwaltungsaktes, ${ }^{*} 9$ mehrstufige Verwaltungsverfahren. ${ }^{*} 80$ VwGO 1993 war nach dem Vorbild ihrer Vorgänger für die Abwehr von belastenden Verwaltungsakten durch die Adressaten geplant. Die Eigentumsreform forderte von Gerichten die Lösung von wesentlich vielfältigeren Situationen: Z.B. wenn ein Grundstück einer ehemaligen Eigentümer zurückgegeben wurde, verlor sein bisherige Nutzer sein Privatisierungsrecht. Dies hat den Staatsgericht dazu gezwungen, Dritte in den Verwaltungsprozess einzubeziehen, obwohl diese Möglichkeit durch VwGO nicht vorgesehen war. ${ }^{* 81}$

\subsection{Reform des allgemeinen Teils des Verwaltungsrechts}

Im Jahr 1999 wurde die neue Redaktion von VwGO verabschiedet und sie ist im Jahr 2000 in Kraft getreten; sie wurde im Kontext der Reform des ganzen allgemeinen Teils des Verwaltungsrechts zusammen mit Entwürfen des Verwaltungsverfahrensgesetzes, des Staatshaftungsgesetzes, des Gesetzes über Ersatzvornahme und Zwangsgeld und des Gesetzes über Verwaltungszusammenarbeit vorbereitet. ${ }^{*} 22$ Mit der Einführung des Gesetzes wurde ein wichtiger Schritt in die Richtung der heutigen Regelung des Verwaltungsprozessrechts

68 §§ 5 und 7 VwGO 1993. Ähnlich auch: § $244^{5}$ ZPO der ESSR.

$69 \S 17$ VwGO 1993; so auch $\S 244^{6}$ Abs. 1 ZPO der ESSR.

$70 \quad \S 11$ VwGO (1993). Nach $\S 244^{6}$ Abs. 2 ZPO der ESSR musste eine Gerichtssache sogar innerhalb von 10 Tagen durchgesehen werden.

$71 \S \S 9$ Abs. 1 Nr. 3 und 4, 14 Abs. 2 und 28 Abs. 4 VwGO. Siehe auch: K. Merusk, I. Koolmeister (Fn. 65), S. 126; K. Merusk. Otsustusmenetlus [Entscheidungsverfahren]. - Juridica 1994/2, S. 54; StGH 16.12.1994, III-3/1-16/94.

$72 \quad$ K. Merusk (Fn. 70), S. 54.

73 StGH hielt diese Betrachtungsweise aus den allgemeinen Grundsätzen des Verwaltungsprozesses abgeleitet, insbesondere StGH 18.6.2002, 3-3-1-33-02, Rdn. 11.

$74 \S 20$ VwGO 1993. So auch § $244^{7}$ Abs. 1 ZPO der ESSR; s. auch StGH 26.5.1995, III-3/1-8/95.

75 K. Merusk, I. Koolmeister (Fn. 65), S. 126.

76 Ü. Madise. Eesti haldusõiguse reformi kandvatest ideedest [Über die tragenden Ideen der Verwaltungsrechtsreform Estlands]. - Juridica 2003/1, S. 38, 42; A. Aedmaa u.a. Haldusmenetluse käsiraamat [Handbuch des Verwaltungsverfahrens]. Tartu: Tartu Ülikooli Kirjastus 2004, S. 34.

77 StGH 6.10.1995, III-3/1-26/95; 10.10.1995, III-3/1-35/95.

78 K. Merusk. Administratsiooni diskretsioon ja selle kohtulik kontroll [Verwaltungsermessen und seine gerichtliche Kontrolle]. Tallinn: Juura 1997, S. 107-114.

79 StGH 13.3.1998, 3-3-1-7-98.

$80 \quad$ StGH 5.12.1997, 3-3-1-32-97, Rdn. 1 .

81 StGH 25.11.1994, III-3/1-11/94.

82 Siehe Ü. Madise (Fn. 75), S. 42-43; Erläuterung zum Gesetzesentwurf von VwGO aus dem Jahr 2000 (VIII Riigikogu 1047 SE), S. 1. 
gemacht. ${ }^{* 83}$ Die Zuständigkeit und die Befugnisse des Verwaltungsgerichtes, sowie der Umfang der gerichtlichen Kontrolle haben genau dann ihre heutige Form erhalten. Auch wurde eine Möglichkeit des schriftlichen Verfahrens geschaffen. Normentechnisch und strukturell blieb das Gesetz doch der Fassung des Jahres 1993 ähnlich, auch der allgemeine Hinweis auf die Bestimmungen der Zivilprozessordnung wurde beibehalten. Parallel zur Erneuerung des Verwaltungsrechts fand eine Reform des Aufbaus der Verwaltungsgerichtsbarkeit statt, die am 1.1.2001 zu Ende gebracht wurde. Alle Verwaltungsrichter der Land- und Stadtgerichte wurden in die Verwaltungsgerichte überführt oder sie setzten ihre Arbeit in bisherigen Gerichten als Richter der ordentlichen Gerichtsbarkeit fort. Die Zuständigkeit der Lösung von Ordnungswidrigkeiten wurde von Verwaltungsgerichten an Land- und Stadtgerichte übergegeben. ${ }^{*} 84$ Mit dieser Veränderung ist in Estland endgültig ein Verwaltungsgerichtssystem herausgebildet worden, das den Traditionen Kontinentaleuropas und dem Sinn von $\S 148$ Abs. 1 Nr. 1 der Verfassung entspricht. ${ }^{*} 85$

Von der Praxis des Staatsgerichts um die Jahrhundertwende müssen insbesondere Urteile hervorgehoben werden, die dem Grundsatz eines lückenlosen und effektiven Rechtsschutzes ${ }^{*} 86$ den Inhalt gegeben haben. Wegen des Fehlens eines andersartigen Rechtsweges wurden verwaltungs-privatrechtlichen Streit igkeiten, ${ }^{*} 7$ Allgemeinverfügungen ${ }^{* 88}$ und unter gewissen Voraussetzungen auch Handlungen des Ermittlungsverfahrens unter die Kontrolle des Verwaltungsgerichts unterzogen. ${ }^{*} 89$ Im Zusammenhang mit der Ergänzung von Befugnissen des Verwaltungsgerichts wurden die Möglichkeiten des Gerichts beim Schützen von Rechten erweitert, aber gleichzeitig wurde dadurch den Klägern die Wahl von richtigen Ansprüchen (Klagearten) erschwert. Das hat zur Ausbildung der Aufklärungspflicht des Gerichts geführt. ${ }^{*} 90$

\section{Hauptmerkmale der estnischen Verwaltungsprozessordnung heute}

Heute werden die Anrufung eines Verwaltungsgerichts und der Ablauf des Verwaltungsgerichtsverfahrens durch die dritte Fassung von VwGO geregelt, die am 1.1.2012 in Kraft getreten ist. Sie basiert hauptsächlich auf VwGO aus dem Jahr 2000 und auf der Kodifizierung ihrer Umsetzungspraxis. Die allgemeine Bezugnahme im $\S 5$ Abs. 1 VwGO a.F. auf Zivilprozessordnung (ZPO) hat sowohl in Gerichten als auch bei Verfahrensbeteiligten einen erheblichen Rechtsunsicherheit verursacht, die im Jahr 2006 durch das Inkrafttreten von neuen detaillierten ZPO noch vertieft wurde. Deshalb werden die Bestimmungen der Zivilprozessordnung in Verwaltungsgerichten jetzt nur in den in neuen VwGO konkret genannten Fällen angewandt. ${ }^{*}{ }^{*}$

\subsection{Aufbau von Verwaltunsgerichten}

In der ersten Instanz werden die Verwaltungssachen in Estland in zwei Verwaltungsgerichten gelöst - in Tallinn und in Tartu. Beide haben eine Außenstelle außerhalb des Hauptgeschäftssitzes - in Pärnu und in Jõhvi. Gegen ein Urteil eines Verwaltungsgerichtes kann ein Rechtsmittel beim Bezirksgericht eingelegt werden, das Urteil des letzteren kann durch eine Revision im höchsten Gericht des Staates - im Staatsgericht - angefochten werden. Die Verwaltungsgerichte sind die von allgemeinen Gerichten organisatorisch getrennte Gerichtsbehörden, dagegen in Bezirksgerichten und im Staatsgericht handeln die

83 Dazu näher unten (2).

84 Ausführungsgesetz der Verwaltungsprozessordnung - RT I 2000, 51, 321; I. Pilving. Halduskohtute korraldus ja pädevus halduskohtumenetluse seadustiku eelnõus [Das System und die Zuständigkeit der Verwaltungsgerichte nach dem Entwurf der Verwaltungsprozessordnung]. - Juridica 1998/10, S. 515.

85 I. Pilving (Fn. 83), S. 515 .

86 z.B. in Verbindung mit dem vorläufigen Rechtsschutz: StGH 21.12.2001, 3-3-1-67-01; StGH der Klagefrist StGH 18.12.2001, 3-3-1-60-01; 15.10.2002, 3-3-1-48-02.

87 StGH 20.12.2001, 3-3-1-15-01.

88 StGH 7.5.2003, 3-3-1-31-03.

89 StGH 22.12.2000, 3-3-1-38-oo (in englischer Sprache: http://www.nc.ee/?id=442).

90 StGH 3.4.2002, 3-3-1-14-02, Rdn. 26.

$91 \S 1$ Abs. 3 VwGO - RT I, 23.12.2013, 38 (Übersetzung ins Englische https://www.riigiteataja.ee/en/eli/527012014001/ consolide). 
Verwaltungskammern in demselben Gerichtsbehörde neben Zivil- und Strafkammern. Alle Verwaltungssachen beginnen im Verwaltungsgericht, u.a. Klagen gegen Einzelentscheidungen der Regierung und Minister. ${ }^{*} 92$

In der ersten Instanz bearbeitet der Richter die Sache in der Regel allein, wegen der Kompliziertheit der Sache oder zur Sicherung der Unabhängigkeit der Rechtsprechung kann ein Spruchkörper von drei Berufsrichtern mit der Lösung der Sache beauftragt werden. ${ }^{*} 3$ Im Jahr 2013 wurden den estnischen Verwaltungsgerichten insgesamt 2957 Klagen eingereicht. Die durchschnittliche Bearbeitungszeit der Verwaltungssachen betrug im Jahr 2013 in der ersten Instanz 144 Tage. Vor allem hat man im letzten Jahr nach einem Rechtsschutz in Bereichen Justizverwaltung (vor allem Klagen von Häftlingen gegen JVA-Verwaltung), Steuerrecht, Baurecht und Sozialrecht gesucht. ${ }^{*} 4$ Der aus 5 Mitgliedern bestehenden Verwaltungskammer des Staatsgerichts wurden im Jahr 2013 insgesamt 652 Anträge eingereicht, 103 wurden zugelassen.

\subsection{Zulässigkeit der Klage}

Die Verwaltungsgerichte sind zuständig für alle öffentlich-rechtlichen Streitigkeiten, für die das Gesetz keinen anderen Rechtsweg vorsieht. Die Zuständigkeit erweitert sich auch auf Steuer- und Sozialrechtssachen, Staatshaftungssachen, öffentliche Ausschreibungen und auf verschiedene zivilrechtliche Geschäfte von öffentlich-rechtlichen juristischen Personen. Das Verwaltungsgericht kann auch zivilrechtliche Tatsachen inzident auswerten, wenn das für die Lösung der Verwaltungsstreitigkeit notwendig ist. ${ }^{*} 5$ Möglich sind Aufhebungsklage, allgemeine Verpflichtungsklage, Unterlassungsklage, Entschädigungsklage in Staatshaftungssachen und Folgenbeseitigungsklage ${ }^{*} 96$ sowie Feststellungsklage. Anfechten kann man sowohl Verwaltungsakten ${ }^{*} 97$ als auch Verträge und Handlungen. ${ }^{*}{ }^{8}$ Unmittelbar können die Verordnungen der Exekutivgewalt in Estland nicht angefochten werden: Möglich ist nur eine konkrete Normenkontrolle über die Verfassungsmäßigkeit solcher Gesetze und Verordnungen, die in einer Streitigkeit über eine Einzelentscheidung relevant sind. Gleichzeitig wird der Begriff des Verwaltungsakts erweitert ausgelegt, z.B. werden Bebauungspläne, Entscheidungen über die Inschutznahme von Naturobjekten ${ }^{*} 99$ und Umweltnutzungspläne ${ }^{* 100}$ als Allgemeinverfügungen für einen Verwaltungsakt gehalten und sie können daher angefochten werden.

Die allgemeine Klagefrist beträgt bei Verwaltungssachen 30 Tage ab der Bekanntgabe einer Verwaltungsentscheidung, bei Schadensersatzsachen 3 Jahren ab dem Auftritt des Schadens. Es ist das Recht zur Erhebung eines Widerspruchs gewährleistet, in der Regel ist die Verwendung eines Vorverfahrens aber nicht verbindlich. Eine Person hat die Klagebefugnis in der Regel nur zum Schutz ihrer subjektiven öffentlichen Rechte. ${ }^{*}{ }^{101}$ Eben der Schutz von Rechten wird im Gesetz als die Hauptaufgabe der Verwaltungsgerichte festgehalten. ${ }^{*} 102$ Aber z.B. in Streitigkeiten über Bebauungspläne ist jedem das Recht auf die Erhebung einer Popularklage gewährleistet. ${ }^{*}{ }^{103}$ In Umweltsachen ist den Umweltverbände die Klagebefug-

92 Nach der Bewertung des Staatsgerichts erlaubt die Verfassung kein Ersetzen einer Gerichtsinstanz durch ein Verfahren in Organen der Exekutivgewalt, StGH 8.6.2009, 3-4-1-7-08, Rdn. 31-33 (in englischer Sprache: http://www.nc.ee/?id=1037); s. auch die Übersicht des Justizsystems in englischer Sprache: http://www.nc.ee/?id=188.

93 Zum Vorhergesagten: $\S \S 3$ und 11 VwGO.

94 Justizministerium Estlands. Die aggregierte Daten der Verfahrensstatistik der Gerichten der I. und II. Instanz aus dem Jahr 2013 (Im Internet verfügbar unter: http://www.kohus.ee/et/eesti-kohtud/kohtute-statistika).

$95 \S 4$ VwGO.

96 E. Andresen. The Claim for Elimination of Unlawful Consequences and the Claim for Compensation for Damage under Estonian State Liability Law. - Juridica International 2005, S. 168.

97 Zur Anfechtung von abgelaufenen Verwaltungsakten siehe E. Vene. Wirksamkeit des angefochtenen Verwaltungsakts als Voraussetzung für die Stattgebung der Aufhebungsklage in der Rechtsetzung und Rechtsprechung Estlands. - Juridica International 2013, S. 142.

98 §§ 5 und 6 VwGO.

99 StGH 31.5.2011, 3-3-1-85-10, Rdn. 20 (Übersetzung ins Englische http://www.riigikohus.ee/?id=1269).

100 I. Pilving. Environmental Exploitation Plan as Administrative Form of Action. - Juridica International 2010, S. 138, $143-144$.

$101 \S \S 44-47$ VwGO. Siehe auch: I. Pilving. Right of Action in Estonian Administrative Procedure. - Juridica International 1999, S. 55 .

102 Zusätzlich hat der Staatsgerichtshof die besondere Rolle der Verwaltungsgerichte beim Ausgleichen der Exekutivgewalt betont - StGH 22.11.2011, 3-3-1-33-11, Rdn. 26.3 (Übersetzung ins Englische http://www.riigikohus.ee/?id=1299); auch aus der historischen Perspektive gesehen: L. Kanger, M. Leppik (Fn. 20), S. 509.

$103 \S 26$ Abs. 1 des Planungsgesetzes. 
nis unabhängig der Verletzung ihrer eigenen subjektiven Rechte oder der subjektiven Rechte von Dritten gewährleistet. ${ }^{* 104}$ Auch eine Einzelperson muss nicht unbedingt in Umweltsachen einen Eingriff in ihre Rechte nennen, ausreichend ist eine sog. erhebliche und tatsächliche Betroffenheit, d.h. eine Verletzung glaubwürdiger Interessen. ${ }^{* 105}$ Neben dem Klagerecht des Einzelnen wurde den Aufsichtsbehörden das traditionelle Recht zum Protest beibehalten. Wie früher, hat die Klage selbst auch heute keine aufschiebende Wirkung auf einen Verwaltungsakt, aber das Gericht kann notfalls die Wirksamkeit des Aktes aufschieben oder andere einstweilige Anordnungen durch einen Beschluss des vorläufigen Rechtsschutzes erteilen. ${ }^{* 106}$

\subsection{Verfahrensgrundsätze und Überprüfungsverfahren der Sache}

Bei der Lösung der Sache muss das Gericht Grenzen des Anspruches und des Klagegrundes einhalten. Das Gericht ist bei der Überprüfung einer Verwaltungsentscheidung nicht an faktische oder rechtliche Aussagen der Beteiligten gebunden. ${ }^{*} 107$ Im Verwaltungsgericht gilt der Untersuchungsgrundsatz: Der Richter muss den Beteiligten, vor allem dem Kläger, beraten und helfen, sollte sie oder er keine Rechtswissen haben. Diese Pflicht gilt sowohl für die Formulierung der Anträge als auch für die Beweiserhebung. ${ }^{*} 108$

Die Ermessensentscheidungen der Verwaltung überprüft das Gericht nach den im Verwaltungsverfahrensgesetz festgehaltenen Ermessensregeln. Der maßgebliche Zeitpunkt für die Bewertung der Rechtmäßigkeit der Verwaltungstätigkeit ist der Moment ihrer Durchführung. ${ }^{*} 109$ Im Rahmen eines Verwaltungsprozesses können Verfahrens- und Formfehler der Verwaltung nicht korrigiert werden, wohl kann ein Fehler unberücksichtigt bleiben, wenn das Gericht überzeugt ist, dass der Fehler keine Auswirkung auf den Inhalt des Verwaltungsaktes hatte. ${ }^{* 110}$

In der Regel muss eine Verwaltungssache mündlich in einer Sitzung besprochen werden, ausnahmsweise sind auch ein schriftliches Verfahren oder ein Verfahren nach billigem Ermessen möglich. Das Gesetz regelt jetzt auch das Mediationsverfahren: Mit der Zustimmung aller Verfahrensbeteiligten kann der Richter den Beteiligten dabei helfen, die Sache einvernehmlich zu lösen. ${ }^{*}{ }^{111}$

\section{Zusammenfassung}

Sowohl am Anfang als auch am Ende des 20. Jahrhunderts ist zunächst das Russische Imperium und später die UdSSR unmittelbar vor der Auflösung und vor der Unabhängigkeit Estlands zur Erkenntnis gekommen, dass die Rechte von Personen zur Zügelung der Exekutive gerichtlich geschützt werden müssen. In beiden Fällen haben eben diese Entwicklungen auch in Estland die gerichtliche Kontrolle der Verwaltung in Gang gesetzt. 1993 ging damit die Bildung von Verwaltungsgerichten als besondere Gerichtsbehörden einher. Das wurde durch das Zusammentreffen von mehreren Tatsachen bedingt: Der politische Wille, eine dem Rechtsstaat eigene gerichtliche Kontrolle über Exekutivgewalt zu gewährleisten, das zeitliche Zusammentreffen der Einführung der Kontrolle mit der Verabschiedung der Gerichtsreform und der Verfassung, der praktische Bedarf die Behandlung der Ordnungswidrigkeiten neu zu gestalten sowie westeuropäische Vorbilder.

Ab AdmGO basiert das Verwaltungsprozessverfahren auf dem Modell des Schutzes vom subjektiven Recht, das durch das Institut des Protestes für den Schutz öffentlicher Interessen ergänzt wurde. Die Zuständigkeit und Befugnisse des Verwaltungsgerichts sind Mal eingeschränkt, dann wieder erweitert worden. Heute sind die Zuständigkeit und Befugnisse des Verwaltungsgerichts größer als je bevor. Allgemein

\footnotetext{
104 K. Relve. Definition of an Environmental Organisation in the Arhus Convention, Environmental Directives and Estonian Law. - Juridica International 2010, S. 121.

105 K. Relve. Influence of Article 9 (3) of the Aarhus Convention on Legal Standing in Estonian Administrative Courts. - Juridica International 2009, S. 176, 180-183.

$106 \S \S 4-6,44,46-47,249,255$ und 292 VwGO.

107 I. Pilving. Der Streitgegenstand im estnischen Verwaltungsprozess. - Juridica International 2009, S. 185, 187.

108 § 2 Abs. 3-5 VwGO.

109 Ab StGH 19.11.2009, 3-3-1-62-09, Rdn. 22 und 7.12.2009, 3-3-1-5-09, Rdn. 44.

$110 \S 158$ Abs. 2-3 VwGO, §§ 4 und 58 des Verwaltungsverfahrensgesetzes. Über Praxis insbesondere: StGH 29.11.2012, 3-3-129-12, Rdn. 19-21.

$111 \S \S 126-141$ VwGO.
} 
verbindliche abstrakte Akten der Verwaltung sind die einzigen verwaltungsrechtlichen Handlungen von Behörden, die nicht unter der Kontrolle der Verwaltungsgerichte fallen. In früheren Perioden hat man versucht, die Tätigkeit der Verwaltungsgerichte wesentlich einzuschränken, das aus der Angst, zu stark ins Tätigkeitsfeld der ordentlichen Gerichte und der Exekutive einzugreifen. Man versuchte die Verwaltungsgerichte z.B. von der Lösung der zivilrechtlichen Fragen und Schadensersatzsachen fernzuhalten. Besonders in den Jahren nach der Wiedererlangung der Unabhängigkeit waren die Befugnisse des Verwaltungsgerichts begrenzt und die Gerichte konnten die Verwaltungsentscheidungen nur im Nachhinein, von der ex post-Betrachtungsweise ausgehend überprüfen, ohne die während des Gerichtsverfahrens sich ändernden Tatsachen und die möglichen neuen Streitigkeiten nach dem Inkrafttreten des Urteils zu berücksichtigen. In letzten 20 Jahren haben die Gesetze und die Rechtsprechung die bisherigen dogmatischen Grenzen andauernd in die Richtung eines effektiveren und kohärenteren Rechtsschutzes verschoben. Die Gerichte sollen nicht nur nachhinein die Rechtmäßigkeit von Verwaltungsentscheidungen einschätzen. Das Ziel ist, den Rechten des Einzelnen einen tatsächlichen Schutz zu bieten, die Aufhebung von Verwaltungsakten nur aus formellen Gründen zu vermeiden und soweit möglich, alle mit einem Fall verbundenen Probleme in einem Verfahren zu lösen (u.a. Schadensersatz, zivilrechtliche Tatsachen und Folgen). 